\title{
MODELO PARAMÉTRICO ANALÍTICO PARA A ESTRUTURA DE VELOCIDADE DO SISTEMA CORRENTE DO BRASIL
}

\author{
André Campos Kersten Schmidt ${ }^{1}$, Wellington Ceccopieri Belo², \\ Ilson Carlos Almeida da Silveira ${ }^{1}$ e José Antonio Moreira Lima² \\ Recebido em 23 novembro, 2006 / Aceito em 14 março, 2007 \\ Received on November 23, 2006 / Accepted on March 14, 2007
}

\begin{abstract}
Velocity-based parametric models are often used in the oceanographic literature either in theoretical studies that involves linear stability properties or as boundary-initial conditions for regional implementations of numerical models. However, most of these models were developed for the Gulf Stream (Niiler \& Robinson, 1967; Luther \& Bane, 1985; Robinson et al., 1988; Arango et al., 1992; Xue \& Mellor, 1993; Gangopadhyay et al., 1997). Because the Brazil Current (BC)- Intermediate Western Boundary Current (IWBC) System is unique in terms of its vertical structure as it flows off SE Brazil, an original parametric model has been developed and is presented in this paper. The model parameterizes both the surface-core structure of the $B C$ as well as the intermediate level-core of the IWBC, keeping their basic features such as the asymmetric configuration due to their interaction with the topography. In the BC case, the model allows for the current shape to be deformed if it is close to the shelf break or to acquire a hyperbolic-like shape when it is positioned far away from the continental margin. In the IWBC parameterization, the main characteristic of the model is to reproduce a core placed at 800-900 $\mathrm{m}$ depth, adjacent to the intermediate portion of the continental slope. Additionally, under the assumption that the currents are in geostrophic balance, a sectional model of the density structure was obtained from the velocity model. This density model was constructed based on the integration of the thermal wind equation. Finally, the parametric model has been successfully tested, it reproduced with great accuracy the structure of three velocity sections obtained from observations within the boundaries of the so-called Campos Bight: $21^{\circ} \mathrm{S}, 22^{\circ} \mathrm{S}$ e $23^{\circ} \mathrm{S}$.
\end{abstract}

Keywords: Brazil Current, Intermediate Western Boundary Current, parametric model.

RESUMO. Modelos paramétricos de velocidade de correntes são utilizados na literatura oceanográfica para estudos teóricos envolvendo propriedades de instabilidade, ou como condições de contorno e iniciais para implementações regionais de modelos numéricos. Estes, no entanto, foram desenvolvidos na sua maioria para a Corrente do Golfo (Niiler \& Robinson, 1967; Luther \& Bane, 1985; Robinson et al., 1988; Arango et al., 1992; Xue \& Mellor, 1993; Gangopadhyay et al., 1997). 0 sistema formado pela Corrente do Brasil (CB) e pela Corrente de Contorno Intermediária (CCI) apresenta características dinâmicas singulares enquanto flui ao largo do SE brasileiro, quando comparada as demais correntes de contorno oeste do oceano mundial. Neste trabalho, apresentamos um modelo paramétrico analítico original. 0 modelo parametriza a estrutura tanto da corrente de superfície (a CB) como da corrente intermediária (a CCI), visando preservar suas características essenciais. No caso da CB, suas principais características são a presença de um núcleo em superfície e o comportamento ocasionalmente assimétrico longitudinalmente devido ao fenômeno do meandramento. Fato que a faz aproximar e afastar o escoamento superficial da quebra de plataforma e do talude superior. Já para a CCI, a principal característica é apresentar o núcleo em torno dos 800-900 m de profundidade, geralmente junto ao talude intermediário. Adicionalmente ao modelo seccional de velocidades, um outro modelo de estrutura de densidade é obtido, assumindo que o campo de velocidades é estacionário e geostrófico: tratam-se, portanto, de campos básicos. Este modelo de densidade é construído a partir da integração da relação do vento térmico. Por fim, a parametrização é confrontada com três seções de velocidades baroclínicas obtidas a partir de observações dentro dos limites da bacia de Campos: $21^{\circ} \mathrm{S}, 22^{\circ} \mathrm{S}$ e $23^{\circ} \mathrm{S}$.

Palavras-chave: Corrente do Brasil, Corrente de Contorno Oeste Intermediária, modelo paramétrico.

\footnotetext{
${ }^{1}$ Instituto Oceanográfico da Universidade de São Paulo (I0-USP), Praça do Oceanográfico, 191, Cidade Universitária, 05508-120 São Paulo, SP, Brasil. Telefone: (11) 3091-6578; Fax: 3091-6610 - E-mails: schmidt@io.usp.br; ilson@io.usp.br

${ }^{2}$ Centro de Pesquisas e Desenvolvimento Leopoldo A. Miguez de Mello, CENPES, PETROBRAS rota 812. Rio de Janeiro, RJ, Brasil. Telefone: (21) 3865-3721

-E-mails: wceccopieri@petrobras.com.br; jamlima@petrobras.com.br
} 


\section{INTRODUÇÃ̃o}

Modelos paramétricos de velocidade de correntes têm sido propostos na literatura oceanográfica seja para estudos teóricos, seja como feições a serem impostas como condições de contorno ou condições iniciais de modelos numéricos. No primeiro caso, são utilizados para comprovação de estrutura dinâmica (ou seja, estudo acerca da composição modal do sistema de correntes) ou na determinação de escoamentos básicos para utilização em modelos de instabilidade geofísica. No entanto, a vasta maioria desses modelos paramétricos foram desenvolvidos para a Corrente do Golfo (Niiler \& Robinson, 1967; Luther \& Bane, 1985; Robinson et al., 1988; Arango et al., 1992; Xue \& Mellor, 1993; Gangopadhyay et al., 1997).

Em contraponto, 0 Sistema Corrente do Brasil (CB) apresenta características dinâmicas singulares quando comparado às demais correntes de contorno oeste e, em particular, à própria Corrente do Golfo (CG). Esta corrente é formada por um escoamento coerente que flui para norte e cujas velocidades decaem monotonicamente com a profundidade (Fig. 1). A CG cresce em transporte com a latitude e atinge mais de $2000 \mathrm{~m}$ de profundidade.

Já 0 Sistema CB (Fig. 2) é composto por um escoamento de superfície para sul (que é a CB propriamente dita) e outro em profundidades intermediárias para norte, comumente designado na literatura como Corrente de Contorno Intermediária (CCI) (Silveira et al., 2000). É nesta forma que o Sistema CB é encontrado ao largo da Margem Continental Brasileira entre $20^{\circ} \mathrm{S}$ e 28S (Boebel et al., 1999), e que será a faixa de latitude de interesse. Ênfase será dada à região da bacia de Campos.

0 padrão de corrente do Sistema CB varia acentuadamente com a latitude. Logo, neste estudo, as funções analíticas construídas para representá-lo serão ajustadas às condições locais, que estão relacionadas a condições topográficas (batimetria) e dinâmicas. Dependendo da localidade, a corrente pode se afastar da quebra de plataforma, inclinar-se e seus núcleos superior (CB) e inferior (CCI) podem alinhar-se ou desalinhar-se, em função da isóbata de ajuste que garanta a conservação de vorticidade potencial. Tais variações também podem estar condicionadas ao meandramento, onde os campos de massa e velocidade se encontram em situação de quase-equilíbrio geostrófico Pode-se, então, esperar que para descrever este sistema de correntes, analítica e seccionalmente, seja necessário ajustar vários parâmetros como: largura, profundidade, eixo, velocidade e inclinação das correntes, para que seja possível representá-lo de acordo com as características capturadas a partir das escassas observações disponíveis.
0 estudo aqui apresentado é a primeira tentativa quali-quantitativa, ao nosso conhecimento, de desenvolver e aplicar uma formulação analítica em balanço geostrófico baseada nas poucas observações existentes do Sistema CB. A parametrização aqui proposta poderá ser utilizada em conjunto com a climatologia para geração de campos iniciais em estudos com modelos numéricos (Gangopadhyay \& Robinson, 2002).

\section{ESTADO BÁSICO DO SISTEMA CB}

Para definir um estado básico que represente de forma adequada a distribuição seccional do Sistema CB na região a ser estudada, necessita-se de fórmulas analíticas que possibilitem preservar as propriedades básicas (médias) da corrente (largura, inclinação, magnitude, transporte, etc.).

Como mencionado anteriormente, no estudo da CG a estrutura da corrente é representada por apenas um núcleo de superfície e sua estrutura decai monotonicamente com a profundidade (Fig. 1). No caso do Sistema CB, na Bacia de Campos, esta é formada por dois núcleos, um de superfície fluindo para sul e outro em nível intermediário, fluindo para norte. Assim sendo, é necessário elaborar uma formulação analítica que permita a representação destes dois núcleos e que reproduza suas características essenciais.

A fórmula analítica construída para reproduzir a estrutura de velocidade básica do Sistema CB foi dividida em dois termos (Fig. 2). 0 primeiro termo caracteriza o núcleo de superfície e será designado $V_{S}(x, z)$; 0 segundo, o núcleo intermediário, será denotado por $V_{i}(x, z)$. De acordo com 0 eixo de coordenadas adotado (Fig. 1) a CB tem direção sul-sudoeste e a CCI norte-nordeste. A representação da estrutura de velocidade total do Sistema CB será dada por

$$
V(x, z)=V_{s}(x, z)+V_{i}(x, z) .
$$

\section{O modelo seccional para a Corrente do Brasil}

A estrutura seccional da corrente de superfície, ou seja, a CB, será descrita por

$$
V_{s}(x, z)=V_{0_{s}} \exp \left\{\frac{z}{z_{s}}\right\}\left\{\begin{array}{c}
\exp \left\{-\left[\frac{\left(x-x_{f} \beta_{c}\right)}{l_{c}}\right]^{2}\right\} \\
\text { se } x \leq x_{f} \\
\exp \left\{-\left[\frac{\left(x-x_{f} \beta_{o}\right)}{l_{o}}\right]^{2}\right\} \\
\text { se } \quad x>x_{f},
\end{array}\right\}
$$

onde $V_{0_{s}}$ é a máxima velocidade do núcleo da corrente em superfície centrada em $x_{f}, z_{s}$ é a taxa de decaimento da velocidade com a profundidade (quanto maior $z_{S}$ menor é a taxa de 




Figura 1 - Representação esquemática de uma seção transversal característica da Corrente do Golfo.

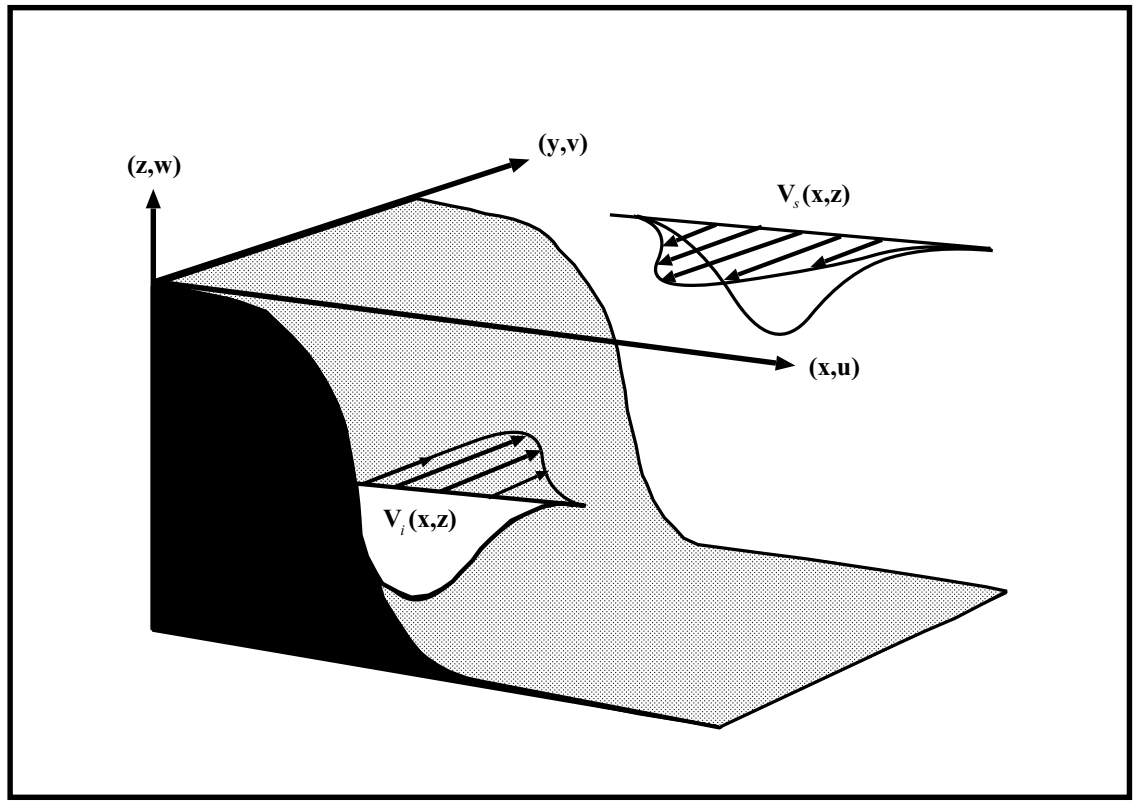

Figura 2 - Representação esquemática de uma seção transversal característica do Sistema Corrente do Brasil. $V_{S}$ indica as velocidades da Corrente do Brasil (CB) e $V_{i}$ a Corrente de Contorno Intermediária (CCI).

decréscimo), $l_{c}$ é a largura da corrente na parte interior (costa), e $l_{o}$, a largura da corrente na parte exterior (oceano). Já

$$
\beta_{c}=1-\frac{z}{z_{s} A_{c}} \quad \text { e } \quad \beta_{o}=1-\frac{z}{z_{s} A_{o}},
$$

representam, respectivamente, a inclinação da corrente nas partes costeira $\left(x \leq x_{f}\right)$ e oceânica $\left(x>x_{f}\right)$ em relação ao eixo vertical $(z)$. $A_{c}$, é 0 parâmetro do grau de inclinação da corrente de superfície na parte costeira, quanto maior $A_{c}$, menor a 
inclinação. Já $A_{o}$ é o parâmetro de inclinação da corrente de superfície na parte oceânica, o qual depende da largura da corrente e da sua inclinação na parte costeira:

$$
A_{o}=A_{c} \frac{l_{c}}{l_{o}} .
$$

Notemos que no caso de $l_{c}=l_{o}$, a corrente é simétrica em relação ao eixo $z$ e $\beta_{c}=\beta_{o}=\beta$. Desta forma, se $\beta=1$, a corrente está alinhada com a vertical (Fig. 3, painel superior), se $\beta>1$ a corrente inclina-se no sentido do oceano aberto (Fig. 3, painel central) e se $\beta<1$, inclina-se no sentido da costa (Fig. 3, painel inferior). No segundo caso observa-se claramente a interação da CB com a quebra de plataforma e talude superior.

\section{0 modelo seccional para a Corrente de Contorno Intermediária}

A estrutura seccional da Corrente de Contorno Intermediária (CCI) é descrita por

$$
V_{i}(x, z)=V_{0_{i}} \exp \left\{\frac{x_{0}-x}{x_{S}}\right\}\left\{\begin{array}{c}
\exp \left\{-\left[\frac{\left(z-z_{f} \gamma_{s}\right)}{h_{s}}\right]^{2}\right\} \\
\text { se } z \leq z_{f} \\
\exp \left\{-\left[\frac{\left(z-z_{f} \gamma_{p}\right)}{h_{p}}\right]^{2}\right\} \\
\text { se } z>z_{f},
\end{array}\right.
$$

onde, $V_{0_{i}}$ é a máxima velocidade no núcleo da corrente em nível intermediário centrada em $x_{0}, z_{f}$ é a posição em profundidade do núcleo, $x_{s}$ é a taxa de decréscimo da velocidade em relação a $x_{0}, h_{s}$ é a espessura da corrente na parte superior, e $h_{p}$ a espessura da corrente na parte inferior.

Os parâmetros

$$
\gamma_{s}=1-\frac{x}{x_{s} B_{s}} \quad \text { e } \quad \gamma_{p}=1-\frac{x}{x_{s} B_{p}},
$$

representam, respectivamente a inclinação da corrente na parte superior e profunda em relação ao eixo horizontal $(x)$. $B_{s}$ é 0 parâmetro do grau de inclinação da corrente intermediária na parte superior $\left(z \geq z_{f}\right)$. Quanto maior $B_{s}$, menor a inclinação. Já $B_{p}$ é 0 parâmetro de inclinação da corrente intermediária na parte profunda $\left(z<z_{f}\right)$, o qual depende da largura da corrente e da sua inclinação na parte superior, na forma dada por

$$
B_{p}=B_{s} \frac{h_{s}}{h_{p}} .
$$

No caso de $h_{s}=h_{p}$, a corrente intermediária é simétrica em relação ao eixo $x$ e $\gamma_{s}=\gamma_{p}=\gamma$. Desta forma, se $\gamma=1$, a corrente está alinhada com 0 eixo horizontal (Fig. 4, painel superior), se $\gamma>1$ a corrente inclina-se no sentido do oceano inferior (Fig. 4, painel central) e se $\gamma<1$, inclina-se no sentido do oceano superior (Fig. 4, painel inferior).

\section{0 modelo seccional para o Sistema CB}

Como colocado anteriormente, a soma da velocidade de superfície $V_{s}(x, z)$ e intermediária $V_{i}(x, z)$ resultará no campo básico do Sistema CB em estudo eq. (1). Assim,

$$
\begin{gathered}
V(x, z)=V_{0_{s}} \exp \left(\frac{z}{z_{s}}\right) \exp \left\{-\left[\frac{\left(x-x_{f} \beta\right)}{l}\right]^{2}\right\} \\
+V_{0_{i}} \exp \left(\frac{x_{0}-x}{x_{S}}\right) \exp \left\{-\left[\frac{\left(z-z_{f} \gamma\right)}{h}\right]^{2}\right\} .
\end{gathered}
$$

Onde a inclinação da corrente é dada por:

$$
\beta=\left\{\begin{array}{l}
\beta_{c} \text { se } x \leq x_{f} \\
\beta_{o} \text { se } x>x_{f}
\end{array} \quad, \gamma=\left\{\begin{array}{l}
\gamma_{s} \text { se } z \geq z_{f} \\
\gamma_{p} \text { se } z<z_{f},
\end{array}\right.\right.
$$

e a largura $(l)$ e espessura $(h)$ por:

$$
l=\left\{\begin{array}{l}
l_{c} \text { se } x \leq x_{f} \\
l_{o} \text { se } x>x_{f},
\end{array} \text { e } h=\left\{\begin{array}{l}
h_{s} \text { se } z \geq z_{f} \\
h_{p} \text { se } z<z_{f} .
\end{array}\right.\right.
$$

Com o intuito de ilustração, foi reconstituído o campo básico (total) resultante (Fig. 5) para os exemplos dos painéis superiores das Figs. 3 e 4, onde a CB é simétrica em relação ao eixo $z$ e a CCl é simétrica em relação ao eixo $x$. Ou seja, trata-se do caso em que $\beta=\gamma=1$.

\section{0 modelo seccional para a estrutura de densidade}

0 objetivo deste trabalho é o desenvolvimento de um modelo paramétrico analítico, cuja estrutura de velocidades represente de fato um estado básico dinamicamente. Assim, busca-se um campo cinemático em balanço geostrófico. Esta imposição permite determinar a estrutura de densidade utilizando a equação do vento térmico (Cushman-Roisin, 1994; Pedlosky, 1987), ou seja:

$$
f \frac{\partial V}{\partial z}=\frac{\partial E}{\partial x} .
$$

onde $f=2 \Omega \operatorname{sen}(\theta)$, $\theta$ é a latitude da seção e $\Omega=$ 7, $29210^{-5}$ rad s$^{-1}$. E representa o termo de empuxo definido por

$$
E=-\frac{g}{\rho_{0}} \rho(x, z),
$$

onde $\rho_{0}$, é a densidade de referência, neste trabalho foi adotado 0 valor de $1023 \mathrm{~kg} \mathrm{~m}^{-3}$. 

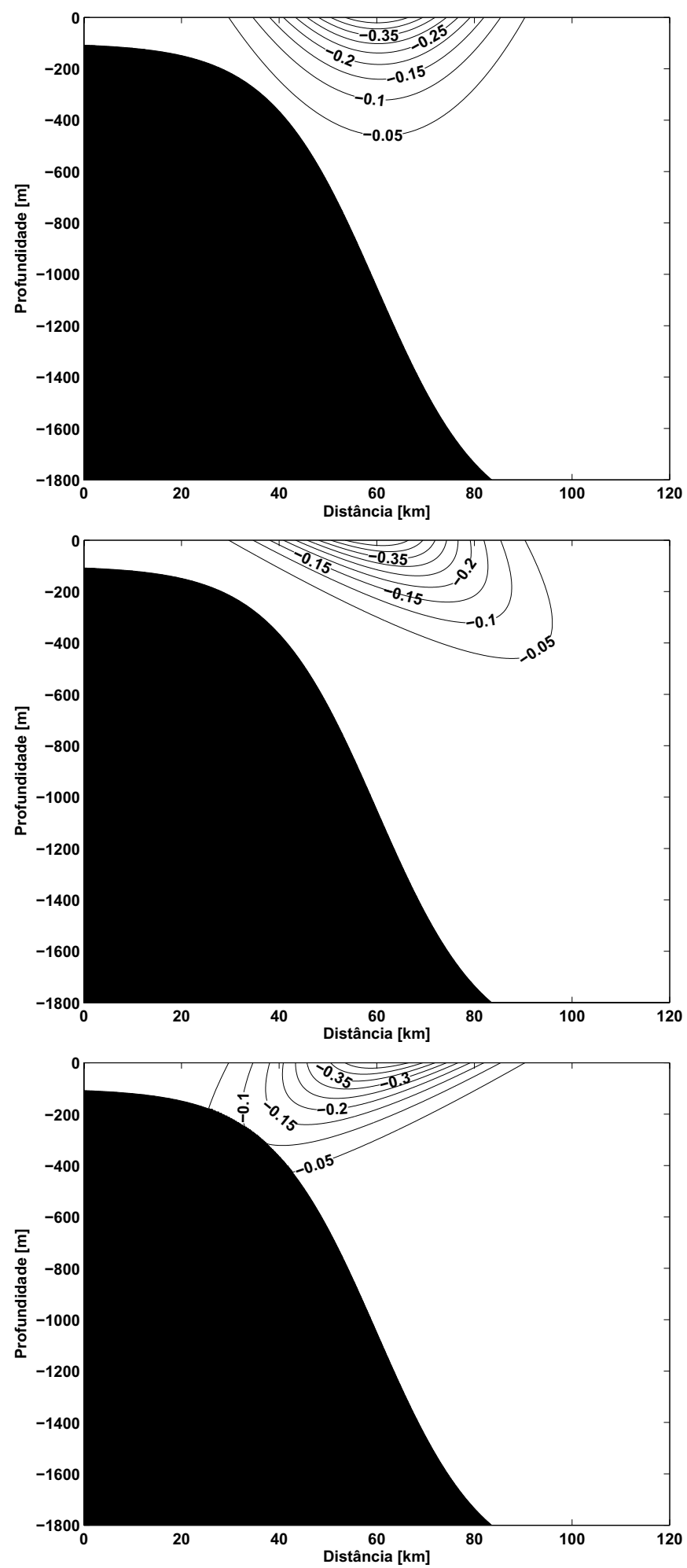

Figura 3 - Seções de velocidade básica para $\beta=1, A_{O}=100$ (painel superior), $\beta>1, A_{O}=5,0$ (painel central) e $\beta<1, A_{o}=-5,0$ (painel inferior). Os parâmetros utilizados para gerar 0 campo de velocidade foram: $V_{0_{s}}=-0,5 \mathrm{~m} \mathrm{~s}^{-1}, l_{c}=l_{o}=20 \mathrm{~km}, x_{f}=60 \mathrm{~km} \mathrm{e} z_{s}=200 \mathrm{~m}$. 

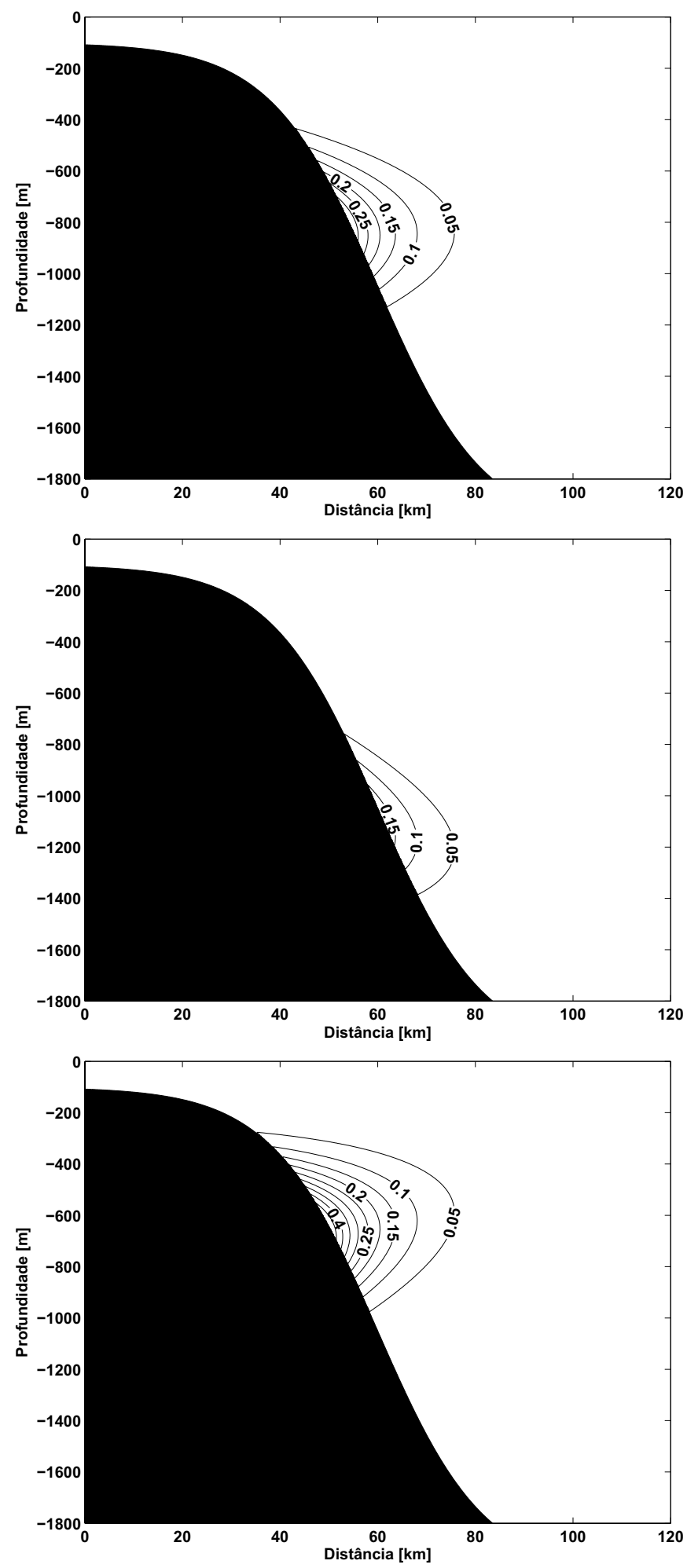

Figura 4 - Seções de velocidade básica para $\gamma=1, B_{p}=100$ (painel superior), $\gamma>1, B_{p}=20$ (painel central) e $\gamma<1, B_{p}=-20$ (painel inferior). Os parâmetros utilizados para gerar 0 campo de velocidade foram: $V_{0_{i}}=0,3 \mathrm{~m} \mathrm{~s}^{-1}, h_{s}=h_{p}=250 \mathrm{~m}, z_{f}=-900 \mathrm{me} x_{s}=11 \mathrm{~km}$. 


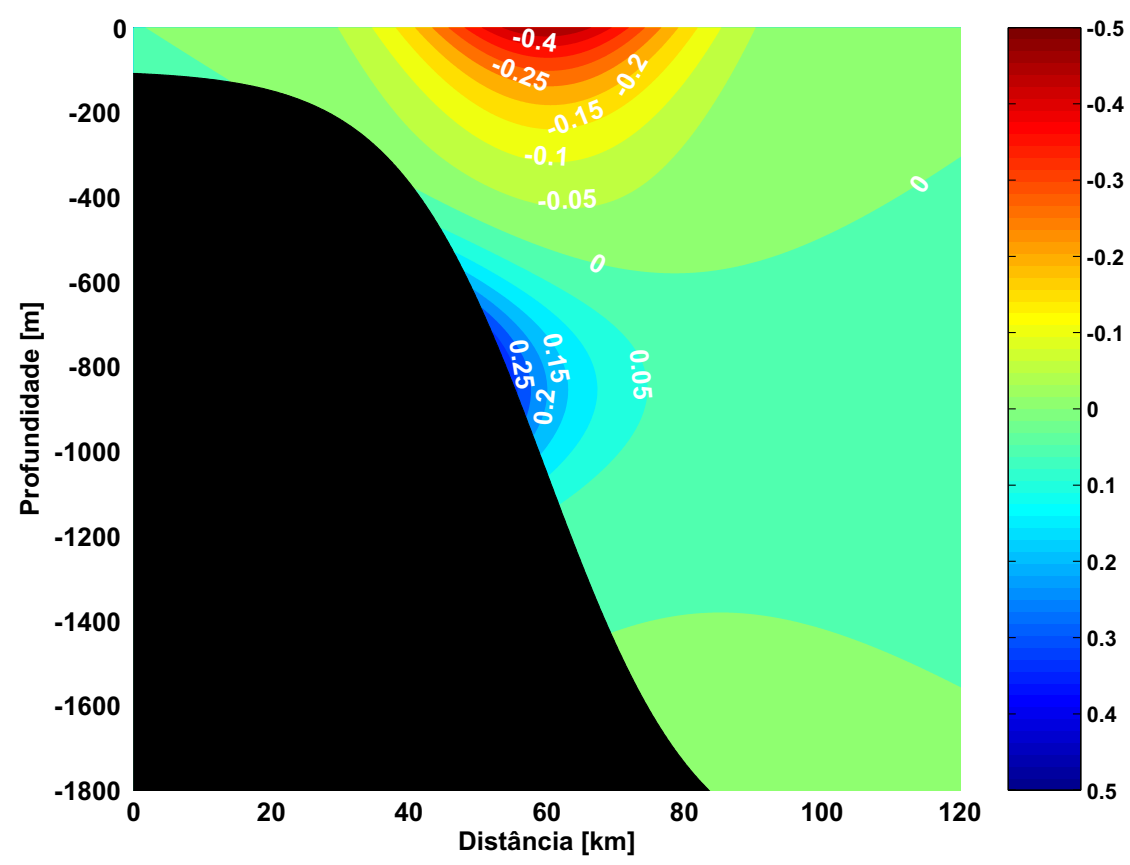

Figura 5 - Seção de velocidade básica para $\beta=\gamma=1, A_{o}=B_{p}=100$. Os parâmetros utilizados para gerar 0 campo de velocidade para a Corrente do Brasil foram: $V_{0_{s}}=-0,5 \mathrm{~m} \mathrm{~s}^{-1}, l_{c}=l_{o}=20 \mathrm{~km}, x_{f}=60 \mathrm{~km}$, $z_{s}=200 \mathrm{~m}$ e para a Corrente de Contorno Intermediária foram: $V_{0_{i}}=0,3 \mathrm{~m} \mathrm{~s}^{-1}, h_{s}=h_{p}=250 \mathrm{~m}$, $z_{f}=-900 \mathrm{me} x_{s}=11 \mathrm{~km}$.

0 gradiente horizontal do campo de empuxo $E_{x}$ é determinado aplicando-se a equação do vento térmico eq. (9) na eq. (10), obtendo-se dessa forma:

$$
\begin{aligned}
E_{x}(x, z)= & \frac{f_{0}}{z_{s}}\left\{1-\frac{2 x_{f}}{A_{0}}\left[\frac{x-x_{f} \beta}{l^{2}}\right]\right\} V_{s} \\
& -2 f_{0} \frac{\left(z-z_{f} \gamma\right)}{h^{2}} V_{i},
\end{aligned}
$$

onde a inclinação da corrente é determinada pela eq. (7) e a largura pela eq. (8).

Para a obtenção do campo de densidade, integra-se a equação do vento térmico eq. (9) em $x$ usando a eq. (10) para chegar a

$$
\rho(x, z)=\rho(0, z)-\frac{f_{0} \rho_{0}}{g} \int_{0}^{L} \frac{\partial V}{\partial z} d x,
$$

onde $L$ é a extensão da seção, $\rho(0, z)$ é o perfil vertical de densidade, o qual pode ser representado pelo perfil médio observado da seção ou por qualquer função analítica que o represente de forma adequada. Neste estudo adotou-se a função linear, $\rho(0, z)=\rho_{0}+c \frac{z}{H_{d}}$, onde $H_{d}$ é a profundidade máxima da seção e $c$ é 0 coeficiente angular determinado a partir de ajuste a dados de densidade disponíveis para a região de estudo.
Aplicando a eq. (11) para 0 exemplo onde $\beta=\gamma=1$ e utilizando $c=4,0$, obtemos 0 campo de densidade. Este campo está representado na Figura 6 em termos de $\sigma_{t}\left(\sigma_{t}=\rho(x, z)-\right.$ 1000).

Como se pode observar na Figura 6, o campo de densidade está de acordo com o campo de velocidade da Figura 5. Isto é, ocorre um soerguimento das isopicnais na porção da CB e um rebaixamento na porção da CCI. 0 campo de densidade é de suma importância na inicialização de modelos numéricos. Em particular, aproximações lineares da equação de estado da água do mar são aplicadas para a obtenção do campo de temperatura potencial e/ou salinidade. Assim sendo, apresentaremos os campos de densidade derivados da eq. (12) para as regiões de estudo.

\section{O modelo seccional para configuração topográfica}

A função utilizada para representar a topografia $h(x)$ desenvolvida por Xue \& Mellor (1993), é escrita em termos da função tangente hiperbólica,

$$
h(x)=H_{s}+\frac{1}{2}\left(H_{d}-H_{s}\right)\left\{1+\operatorname{tgh} \frac{x-x_{m}}{\alpha}\right\},
$$




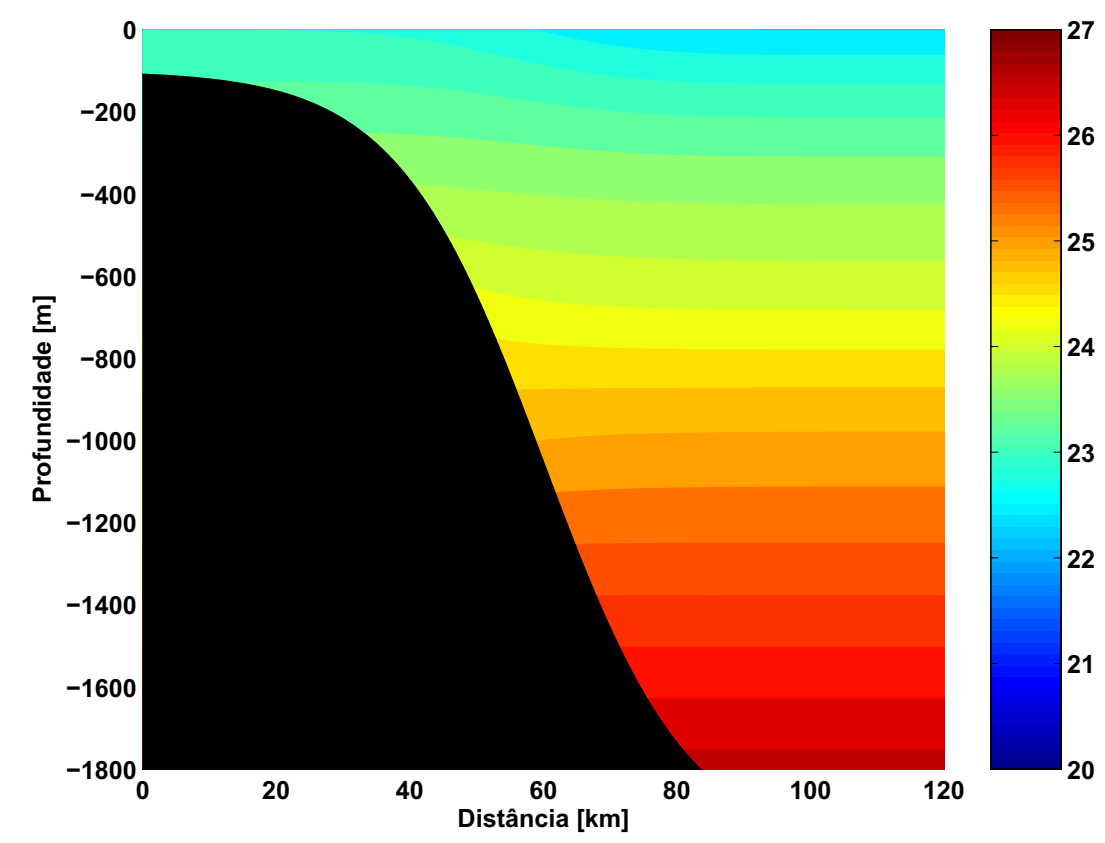

Figura 6 - Seção de densidade básica associada ao campo de velocidade da Figura 5 obtida através das eqs. (11) e (12) para $\beta=\gamma=1, A_{o}=B_{S}=100$ e $c=4,0$.

onde $H_{S}$ é a profundidade média da plataforma. A inclinação do talude é representada por $\alpha$, e $x_{m}$ é a localização da máxima inclinação do talude, em quilômetros. Conforme veremos na próxima seção, os parâmetros utilizados nas fórmulas analíticas são determinados de maneira a se ajustarem aos dados reais disponíveis, embora também possam ser utilizados de forma arbitrária, em estudos de processos dinâmicos do ponto de vista teórico.

\section{APLICAÇÃO DO MODELO PARAMÉTRICO}

Nesta seção serão apresentados os campos básicos do Sistema CB para as latitudes de $21^{\circ} \mathrm{S}, 22^{\circ} \mathrm{S}$ e $23^{\circ} \mathrm{S}$ (Fig. 7), ou seja, nas porções norte, central e sul da Bacia de Campos, RJ.

Serão utilizadas tanto informações já constantes da literatura oceanográfica, quanto dados observacionais disponíveis para a área. Assim, os campos característicos serão obtidos através da aplicação da formulação constante das equações para a velocidade eq. (10) e para a estrutura de densidade básica associada ao escoamento eq. (12).

0 objetivo desta seção não é uma reprodução exata das observações ou um ajuste perfeito por mínimos quadrados, mas sim a reprodução das principais características deste sistema de correntes. Estes campos básicos poderão, no futuro, ser utilizados no estudo analítico de instabilidade ou como condições de contorno e/ou iniciais em implementações regionais de modelos numéricos.

Tabela 1 - Parâmetros utilizados na aproximação analítica da topografia nas seções em $21^{\circ} \mathrm{S}, 22^{\circ} \mathrm{S}$ e $23^{\circ} \mathrm{S}$.

\begin{tabular}{|c|c|c|c|c|}
\hline $\begin{array}{c}\text { Seções } \\
\text { transversais }\end{array}$ & $H_{S}(\mathrm{~m})$ & $H_{d}(\mathrm{~m})$ & $\alpha(\mathrm{km})$ & $x_{m}(\mathrm{~km})$ \\
\hline $21^{\circ} \mathrm{S}$ & 50 & 2000 & 6 & 20 \\
\hline $22^{\circ} \mathrm{S}$ & 100 & 2000 & 22 & 60 \\
\hline $23^{\circ} \mathrm{S}$ & 100 & 1780 & 25 & 72 \\
\hline
\end{tabular}

\section{Latitude de $21^{\circ} \mathrm{S}$}

Os parâmetros utilizados para a construção da topografia característica de uma radial aproximadamente zonal em $21^{\circ} \mathrm{S}$ são apresentados na Tabela 1 e representados na Figura 8. Esta região representa o limite entre o sul da Bacia do Espírito Santo e o norte da Bacia de Campos.

0 ajuste realizado na topografia utilizando-se a função tangente hiperbólica (eq. 13) representa adequadamente a batimetria real até profundidade de aproximadamente 1200 metros. Além desta profundidade, a representação não é adequada. A adição de funções gaussianas seriam necessárias para tal. Porém, como a maior parte da dinâmica do Sistema CB está confinada aos primeiros 1200 metros superiores da coluna de água, optamos por adotar esta representação. 


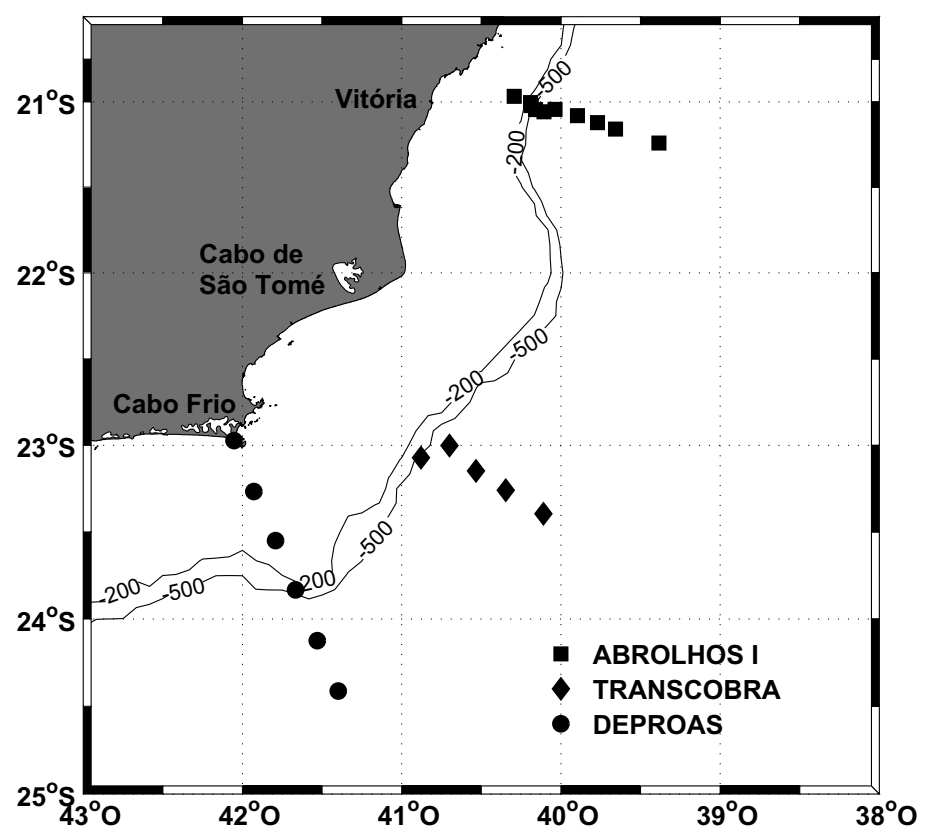

Figura 7 - Posições das radiais dos experimentos ABROLHOSI (setembro de 2004), TRANSCOBRA (abril de 1983) e DEPROAS (julho de 2001).

Tabela 2 - Parâmetros utilizados para gerar os campos básicos de velocidade das seções transversais em $21^{\circ} \mathrm{S}$, $22^{\circ} \mathrm{S}$ e $23^{\circ} \mathrm{S}$ através das eqs. (6), (7) e (8).

\begin{tabular}{|c|c|c|c|c|c|c|c|}
\hline Superfície & $x_{f}(\mathrm{~km})$ & $l_{c}(\mathrm{~km})$ & $l_{o}(\mathrm{~km})$ & $V_{0_{s}}\left(\mathrm{~m} \mathrm{~s}^{-1}\right)$ & $A_{O}$ & $z_{S}(\mathrm{~m})$ & \\
\hline $21^{\circ} \mathrm{S}$ & 45 & 20 & 15 & 0,83 & 20,0 & 135 & \\
\hline $22^{\circ} \mathrm{S}$ & 60 & 25 & 20 & 0,5 & 10,0 & 150 & \\
\hline $23^{\circ} \mathrm{S}$ & 70 & 23 & 23 & 0,6 & 40,0 & 135 & \\
\hline
\end{tabular}

\begin{tabular}{|c|c|c|c|c|c|c|c|}
\hline Intermediária & $z_{f}(\mathrm{~m})$ & $h_{S}(\mathrm{~m})$ & $h_{p}(\mathrm{~m})$ & $V_{0_{i}}\left(\mathrm{~m} \mathrm{~s}^{-1}\right)$ & $B_{p}$ & $x_{S}(\mathrm{~km})$ & $x_{0}(\mathrm{~km})$ \\
\hline $21^{\circ} \mathrm{S}$ & -900 & -250 & -1000 & $-0,24$ & 20,0 & 14 & 20 \\
\hline $22^{\circ} \mathrm{S}$ & -900 & -225 & -650 & $-0,45$ & 20,0 & 8 & 56 \\
\hline $23^{\circ} \mathrm{S}$ & -900 & -200 & -1000 & $-0,45$ & 20,0 & 17 & 65 \\
\hline
\end{tabular}

Os parâmetros utilizados para a construção dos campos de velocidade e densidade para a seção em $21^{\circ} \mathrm{S}$, estão apresentados na Tabela 2. Estes parâmetros foram escolhidos de forma a reproduzir as características dinâmicas e geométricas do Sistema CB na seção de $21^{\circ} \mathrm{S}$. Para reproduzir 0 campo básico de velocidade foram utilizadas informações dinâmicas derivadas da literatura como por exemplo, a velocidade máxima no núcleo da $\mathrm{CB}$, da $\mathrm{CCl}$ e seus respectivos transportes. Neste caso particular, foram utilizados os dados hidrográficos do Cruzeiro ABROLHOS I (setembro de 2004) para gerar um campo de velocidades baroclínicas absolutas, seguindo a metodologia descrita por Silveira et al. (2004). Estas velocidades, exibidas na Figura 9 , não são totalmente geostróficas visto que o modelo empregado (versão seccional do Princeton Ocean Model-POMsec) emprega equações primitivas e condições de não-escorregamento junto aos contornos sólidos. Já o escoamento geostrófico não satisfaz essa condição e o modelo analítico aqui desenvolvido prevê máximos de velocidade junto ao talude.

Na seção de velocidade da Figura 9, observa-se que as velocidades máximas em superfície da CB foram de $-0,83 \mathrm{~m} \mathrm{~s}^{-1} \mathrm{e}$ a velocidade máxima da $\mathrm{CCl}$ de $0,24 \mathrm{~m} \mathrm{~s}^{-1}$. Os transportes associados à CB foram de $-6,7$ Sv e da CCI de 6,0 Sv. 0s parâme- 


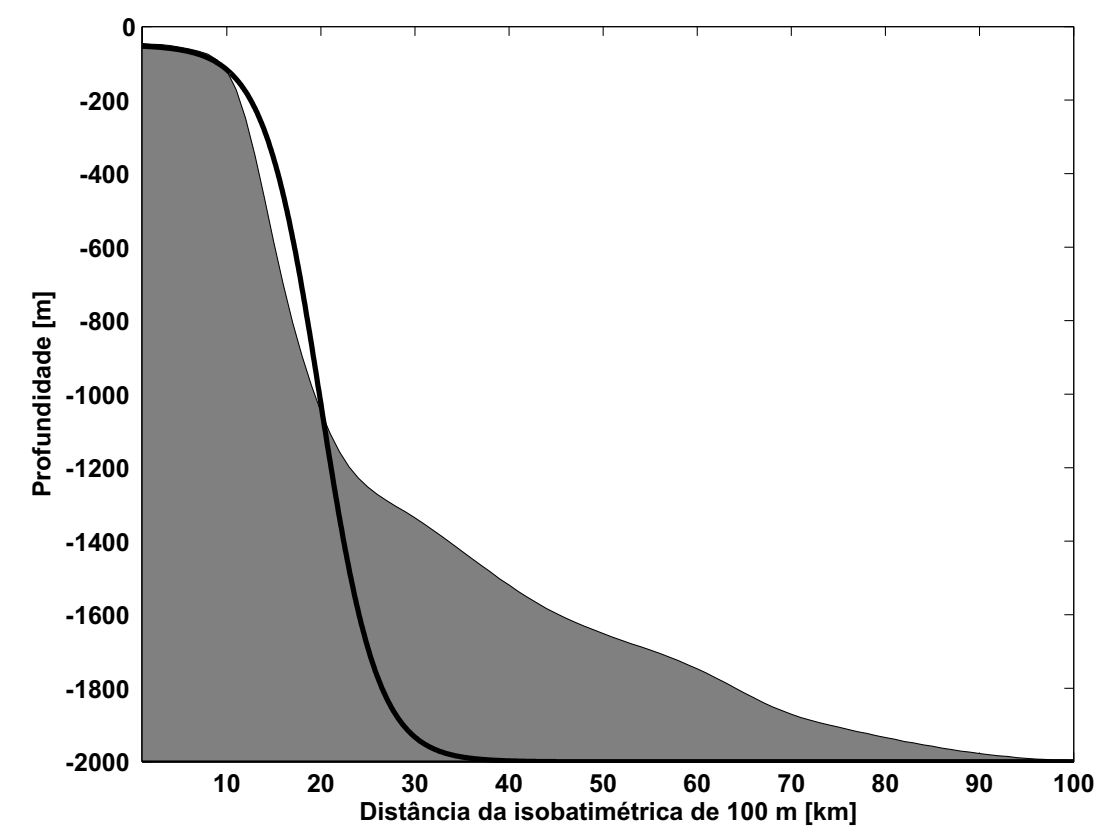

Figura 8 - Topografia real extraída dos dados do Cruzeiro ABROLHOS I (área em cinza) e sua aproximação analítica (linha negra) na seção em $21^{\circ} \mathrm{S}$.

tros foram escolhidos de forma a representar as dimensões das estruturas presentes na Figura 9, e se encontram listados na Tabela 2. 0 campo de velocidade básico (painel superior) e o campo de densidade (painel inferior) obtidos são, respectivamente, apresentados na Figura 10. A CB parametrizada é assimétrica, com 0 lado costeiro de menores dimensões que o lado oceânico, o que reproduz a interação com a quebra de plataforma. Já a CCI atinge máximos de velocidade comparáveis à original, mas o núcleo se posiciona junto ao talude. Os transportes calculados para a CB parametrizada é de -6,79 Sv e, 5,99 Sv para a CCl.

\section{Latitude de $22^{\circ} \mathrm{S}$}

Em seguida o modelo foi aplicado à porção mais central da Bacia de Campos. Os parâmetros utilizados para a construção da topografia característica da região são apresentados na Tabela 1 e o perfil batimétrico representado na Figura 11. Como pode-se observar, 0 ajuste da topografia utilizando-se a função tangente hiperbólica (eq. 13) representa satisfatoriamente a batimetria real até a profundidade de 1800 metros.

Para este exemplo, foram utilizados dados do experimento TRANSCOBRA (Silveira et al., 2004). A seção de velocidades (Fig. 12) mostra que as velocidades máximas em superfície da $\mathrm{CB}$ foram de $-0,5 \mathrm{~m} \mathrm{~s}^{-1}$ e a velocidade máxima da $\mathrm{CCl}$ de $0,5 \mathrm{~m} \mathrm{~s}^{-1}$. Os transportes associados à CB foram de $-5,6 \mathrm{~Sv}$ e, à CCI de 3,6 Sv. Novamente os parâmetros do modelo analítico foram escolhidos com base nos valores de velocidade, dimensões e geometria tanto da CB como da CCI derivados da literatura (Tab. 2).

0 campo de velocidade básico obtido utilizando-se os parâmetros da Tabela 2 são apresentados na Figura 13 (painel superior). Visivelmente, o campo de velocidades parametrizado é bastante mais próximo ao mapeado pelas observações para esta latitude do que para a latitude de $21^{\circ} \mathrm{S}$. Tal concordância é particularmente positiva no domínio da CCI. Em termos de transporte, foram estimados transportes de $-5,7$ Sv para a CB e 3,55 Sv para a CCl. Os valores estimados são muito próximos aos derivados das observações. 0 campo de densidade associado ao campo de velocidade básico é apresentado na Figura 13 (painel inferior).

\section{Latitude de $23^{\circ} \mathrm{S}$}

Os parâmetros utilizados para a construção da topografia característica desta latitude ao largo da costa sudeste brasileira são apresentados na Tabela $1 \mathrm{e}$ o perfil topográfico é representado na Figura 14. Como no caso anterior para $22^{\circ} \mathrm{S}$, 0 ajuste da topografia, utilizando-se a função tangente hiperbólica (eq. 13), aproxima corretamente a batimetria real até a profundidade de 1800 metros. Esta seção está localizada nas proximidades de Cabo Frio, extremo sul da Bacia de Campos. 


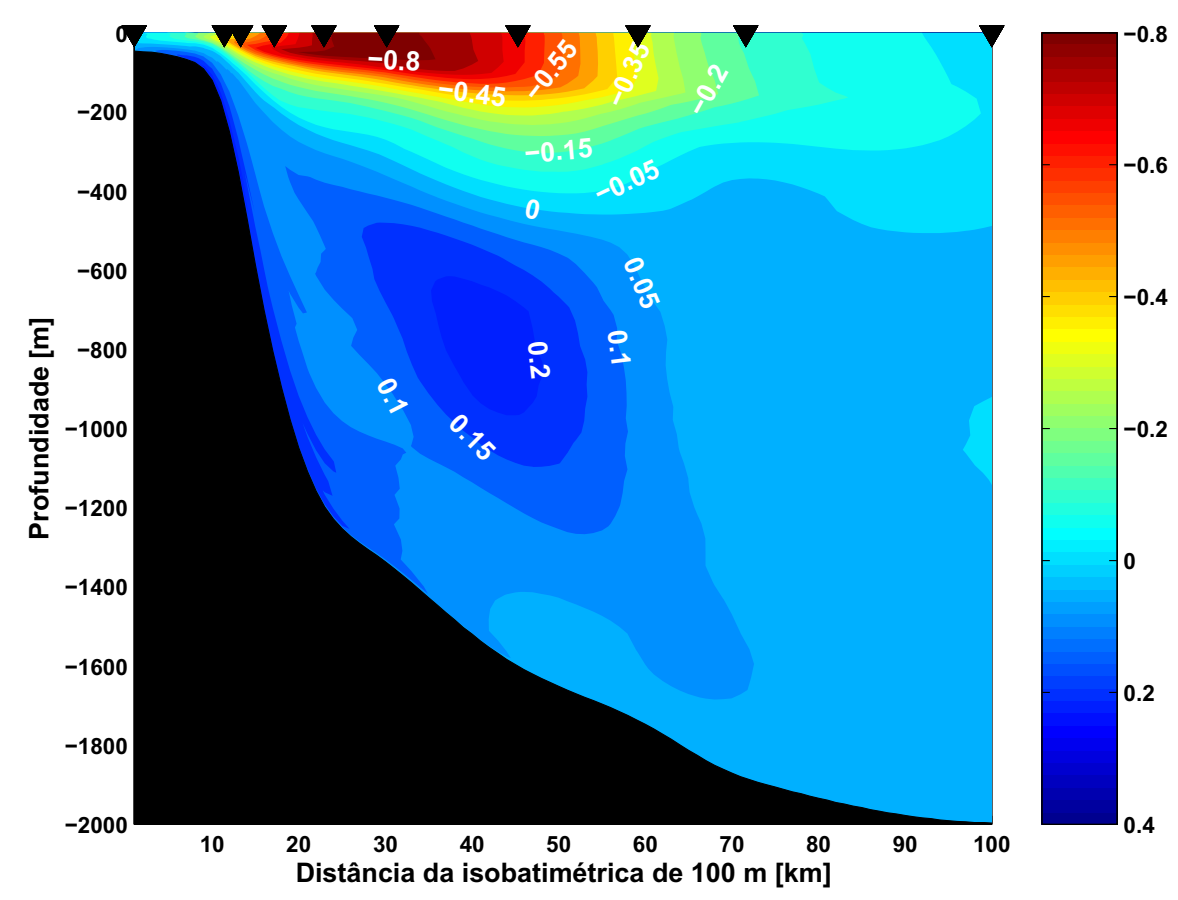

Figura 9 - Seção vertical da velocidade baroclínica absoluta $\left(\mathrm{m} \mathrm{s}^{-1}\right)$ do Cruzeiro ABROLHOS I (setembro de 2004), localizada em $21^{\circ} \mathrm{S}$. Este campo foi obtido após 10 dias de simulação com o modelo POMsec, inicializado com uma grade interpolada a partir de perfis de CTD. Velocidades negativas têm a direção sul-sudoeste e positivas, norte-nordeste.

Neste caso, novamente foram utilizados os resultados de (Silveira et al., 2004); a seção de velocidade obtida está representada na Figura 15. Trata-se de um campo de velocidades baroclínicas absolutas simulado pelo POMsec a partir de perfis de CTD coletados durante o Projeto DEPROAS (julho de 2001).

Os parâmetros utilizados para a construção dos campos de velocidade e densidade para a seção em $23^{\circ} \mathrm{S}$, estão apresentados na Tabela 2 e seguiram os mesmos critérios dos dois casos apresentados anteriormente.

A configuração do campo é semelhante à da seção em $22^{\circ} \mathrm{S}$, com a CCl apresentando núcleo próximo ao talude. Já a CB, está mais distante da quebra de plataforma e apresenta um aspecto mais simétrico que os anteriores. Nesta seção, as velocidades máximas em superfície da CB foram de $-0,6 \mathrm{~m} \mathrm{~s}^{-1}$ e a velocidade máxima da $\mathrm{CCl}$ de $0,32 \mathrm{~m} \mathrm{~s}^{-1}$. Os transportes associados à CB foram de -7 Sv e da CCI de 7 Sv. 0 campo de velocidade parametrizado é apresentado na Figura 16. Foram estimados transportes de $-6,48$ Sv para a CB e de 6,16 Sv para a CCI.

\section{CONSIDERAÇÕES FINAIS}

Um modelo paramétrico, de cunho analítico, foi formulado para o Sistema CB ao largo do sudeste brasileiro. 0 modelo parametriza a estrutura tanto da corrente de superfície (a CB) como da corrente intermediária (a CCI), visando preservar suas características essenciais. No caso da CB, distingui-se o núcleo em superfície e o comportamento ocasionalmente assimétrico longitudinalmente devido ao fenômeno do meandramento, que aproxima e afasta 0 escoamento da quebra de plataforma e do talude superior. Já para a CCl, a principal característica é apresentar o núcleo em torno dos 800-900 m de profundidade, geralmente junto ao talude intermediário.

A estrutura de densidade foi obtida assumindo-se que 0 campo de velocidades é estacionário e geostrófico: tratam-se de campos básicos. A integração da relação do vento térmico permite a composição seccional da estrutura de densidade. A topografia da seção também é parametrizada seguindo Xue \& Mellor (1993).

0 modelo paramétrico foi testado para três seções de velocidade nos limites da Bacia de Campos (RJ): $21^{\circ} \mathrm{S}, 22^{\circ} \mathrm{S}$ e $23^{\circ} \mathrm{S}$. Destas três seções, duas $\left(22^{\circ} \mathrm{S}\right.$ e $\left.23^{\circ} \mathrm{S}\right)$ foram apresentadas por Silveira et al. (2004). A de $21^{\circ} \mathrm{S}$ foi calculada a partir de dados de CTD originais do Projeto ABROLHOS, seguindo a metodologia dos autores supracitados.

Para os três casos, os vários parâmetros do modelo analítico foram escolhidos criteriosamente considerando-se velocidades 

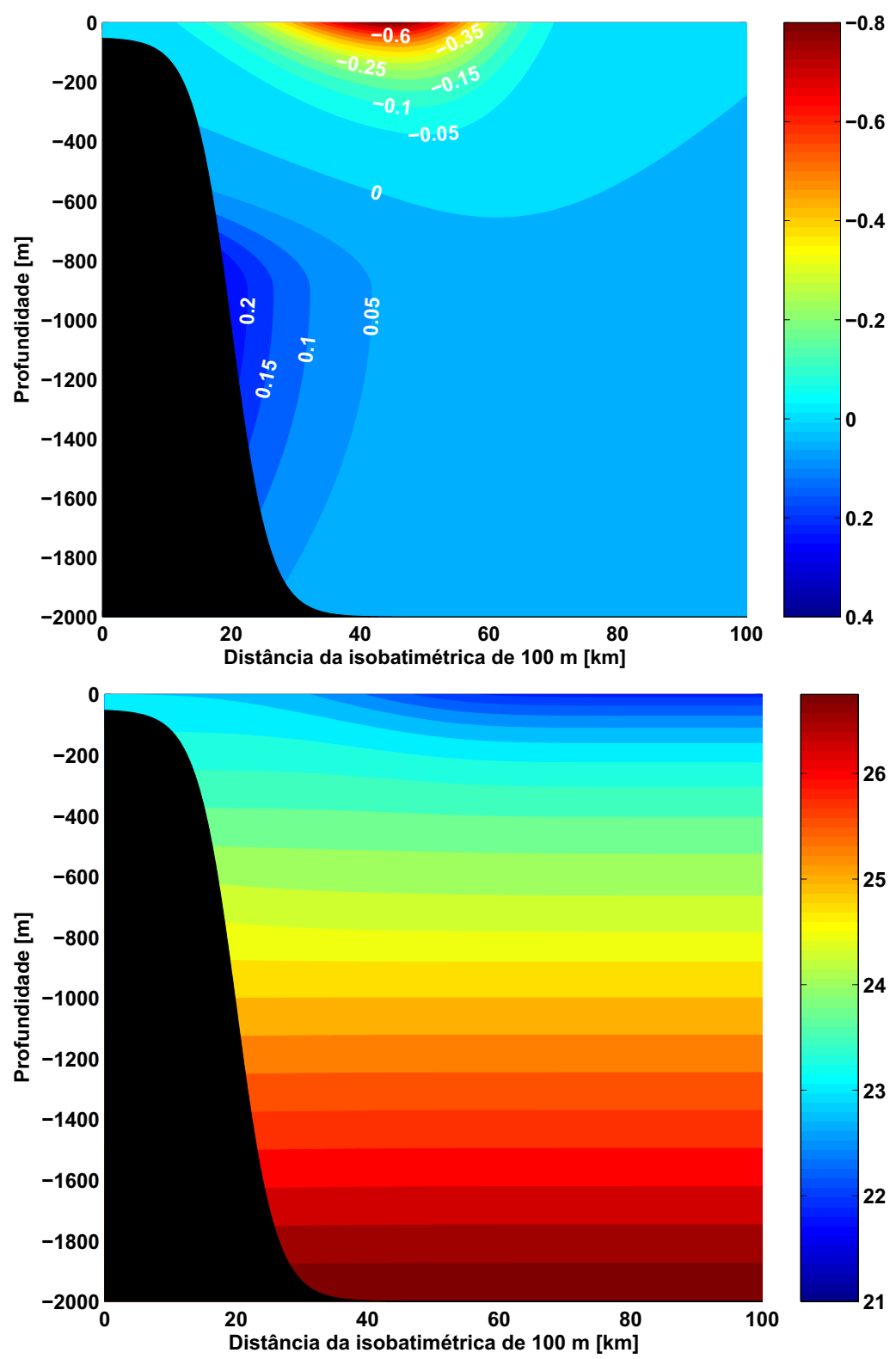

Figura 10 - Estado básico do Sistema CB, representativo de uma seção transversal na latitude de $21^{\circ} \mathrm{S}$ : campo de velocidade geostrófica em m s${ }^{-1}$ (painel superior) e campo de densidade $\sigma_{t} \mathrm{em} \mathrm{kg} \mathrm{m}^{-3}, c=4,0$ (painel inferior). Velocidades negativas têm a direção sul-sudoeste, e positivas norte-nordeste.

máximas, posição e geometria das correntes. Os resultados mostraram-se bastante satisfatórios em termos de transporte de volume, com erros não maiores que $10 \%$. Por outro lado, as configurações do escoamento foram bastante realistas para as radiais de $22^{\circ} \mathrm{S}$ e $23^{\circ} \mathrm{S}$, embora a geometria da $\mathrm{CCl}$ não tenha sido corretamente reproduzida para a latitude de $21^{\circ} \mathrm{S}$. Podem ser apontadas razões para esta discordância. A primeira é que a altura de $21^{\circ} \mathrm{S}$, é sabido que a CCI começa a se afastar do ta- lude para contornar a Cadeia Vitória-Trindade e rumar para a costa leste (Stramma \& England, 1999). A segunda é que, assim como ocorre com a $\mathrm{CB}$, é possível que a $\mathrm{CCl}$ meandre e seu núcleo também deixe as imediações do talude. 0 modelo paramétrico aqui desenvolvido e aplicado estabelece que o núcleo da $\mathrm{CCl}$ é sempre encontrado nas proximidades do talude intermediário, conforme as únicas observações diretas disponíveis de Boebel et al. (1999) e as seções de Silveira et al. (2004). 


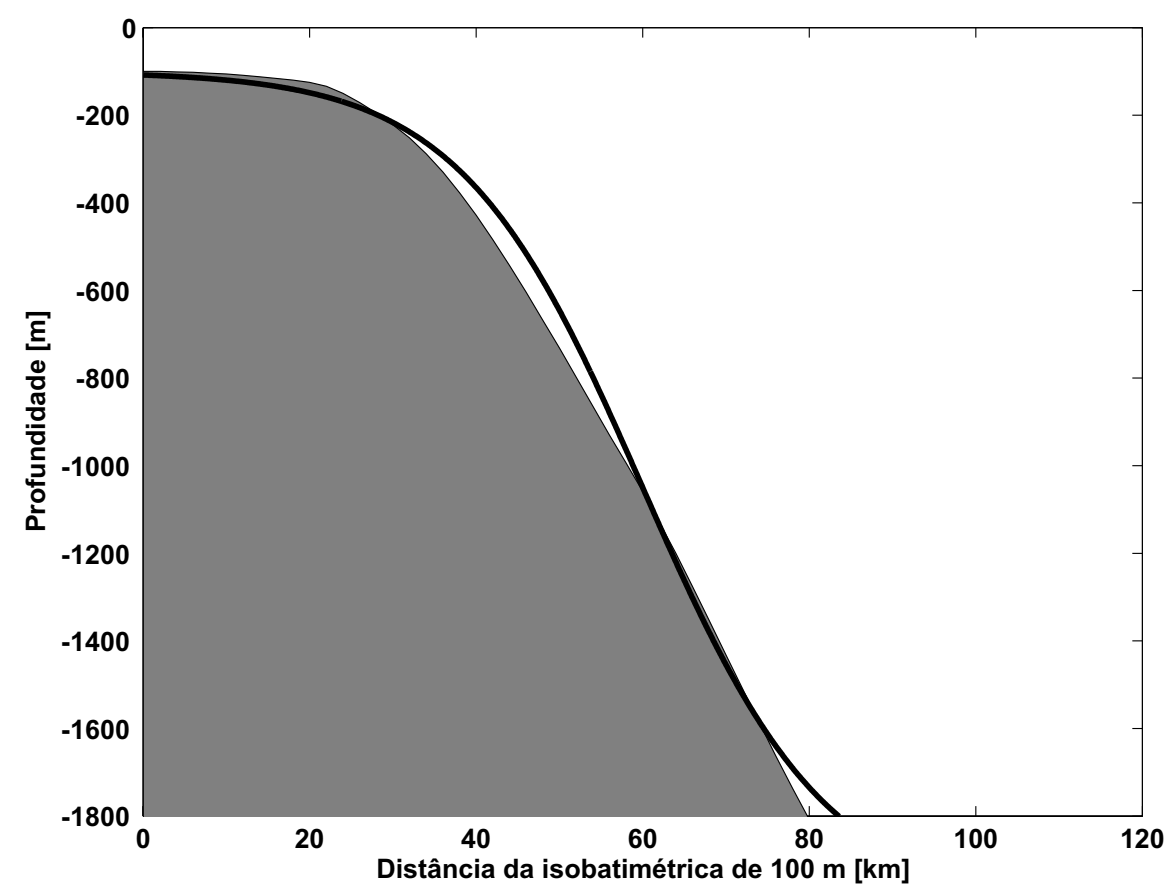

Figura 11 - Topografia real extraída dos dados do Cruzeiro TRANSCOBRA (área em cinza) e sua aproximação analítica (linha negra) na seção em $22^{\circ} \mathrm{S}$.

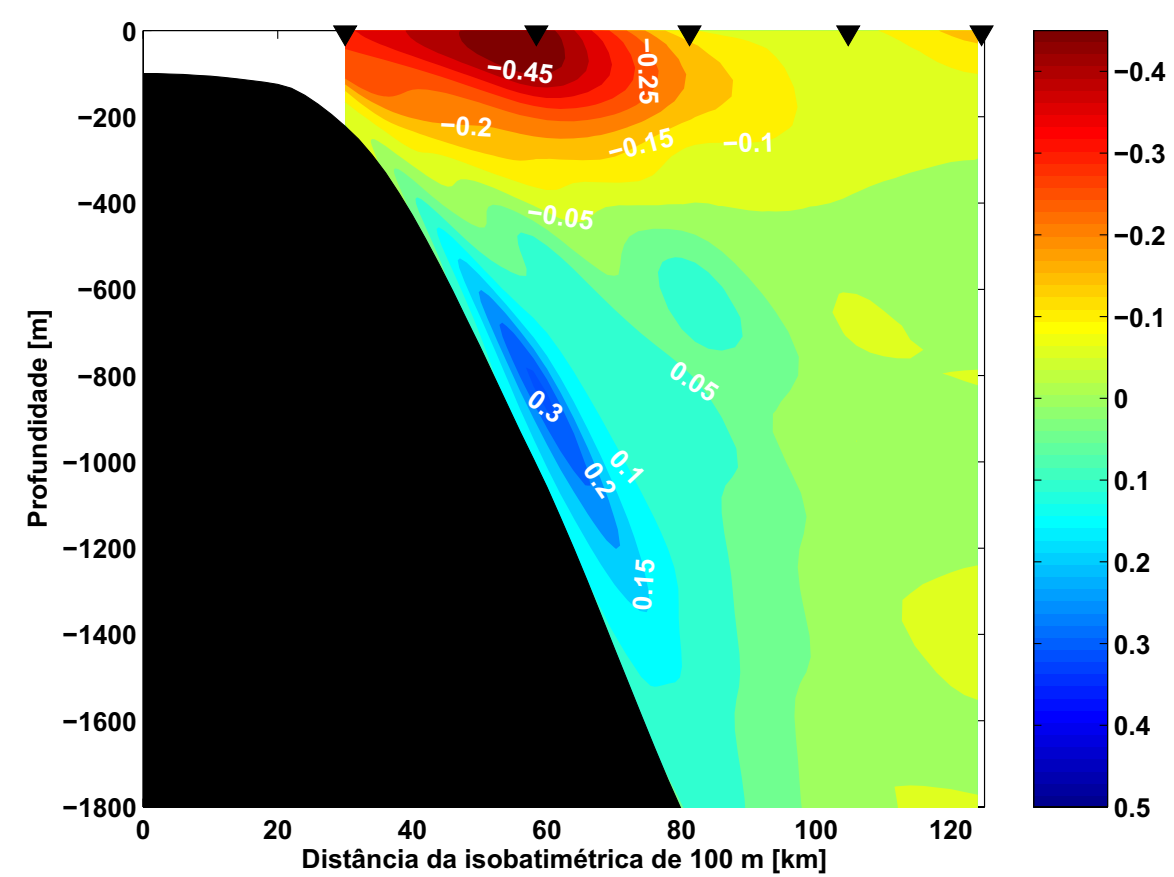

Figura 12 - Seção de velocidade observada em $\mathrm{m} \mathrm{s}^{-1}$ durante o experimento TRANSCOBRA em $22^{\circ} \mathrm{S}$, em abril de 1983 (Silveira et al., 2004). Velocidades negativas têm a direção sul-sudoeste, e positivas norte-nordeste. 

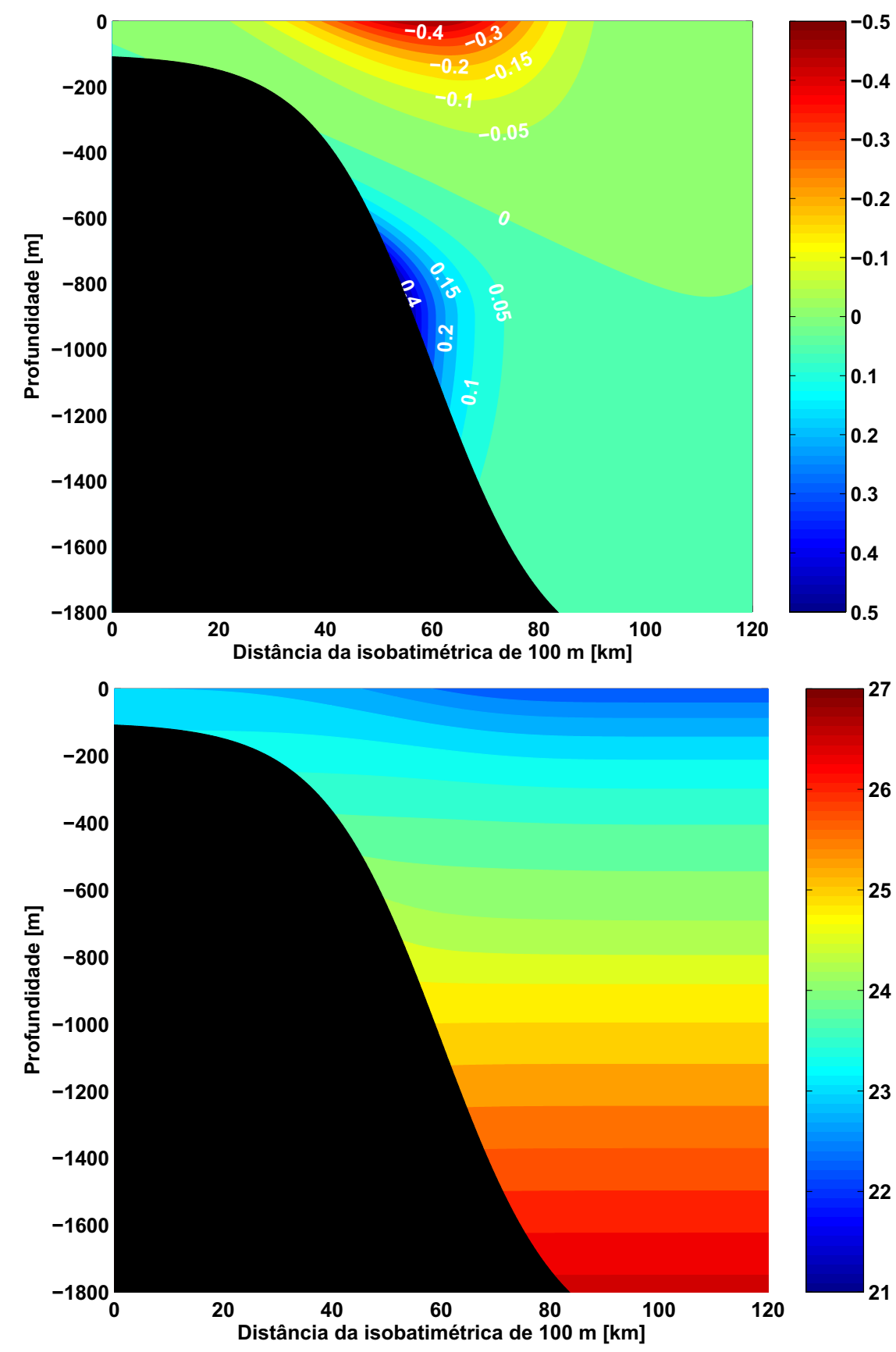

Figura 13 - Estado básico do Sistema CB, representativo de uma seção transversal na latitude de $22^{\circ} \mathrm{S}$ : campo de velocidade geostrófica em $\mathrm{m} \mathrm{s}^{-1}$ (painel superior) e campo de densidade $\sigma_{t} \mathrm{em} \mathrm{kg} \mathrm{m}^{-3}$ (painel inferior). Velocidades negativas têm a direção sul-sudoeste, e positivas norte-nordeste.

Deve ficar claro ao leitor que o modelo paramétrico aqui apresentado representa a primeira tentativa de descrever 0 Sistema CB por funções matemáticas. Encontra-se em elaboração uma versão mais aprimorada que permite o "descolamento" do núcleo da $\mathrm{CCl}$ em latitudes mais tropicais. Entretanto, o modelo paramétrico na presente forma pode ser utilizado como forma de incluir estrutura de meso-escala em implementações de modelos numéricos regionais. Caso se deseje obter os campos de temperatura associados aos campos básicos paramétricos, sugere-se que seja seguida a metodologia proposta por Fernandes (2005), 


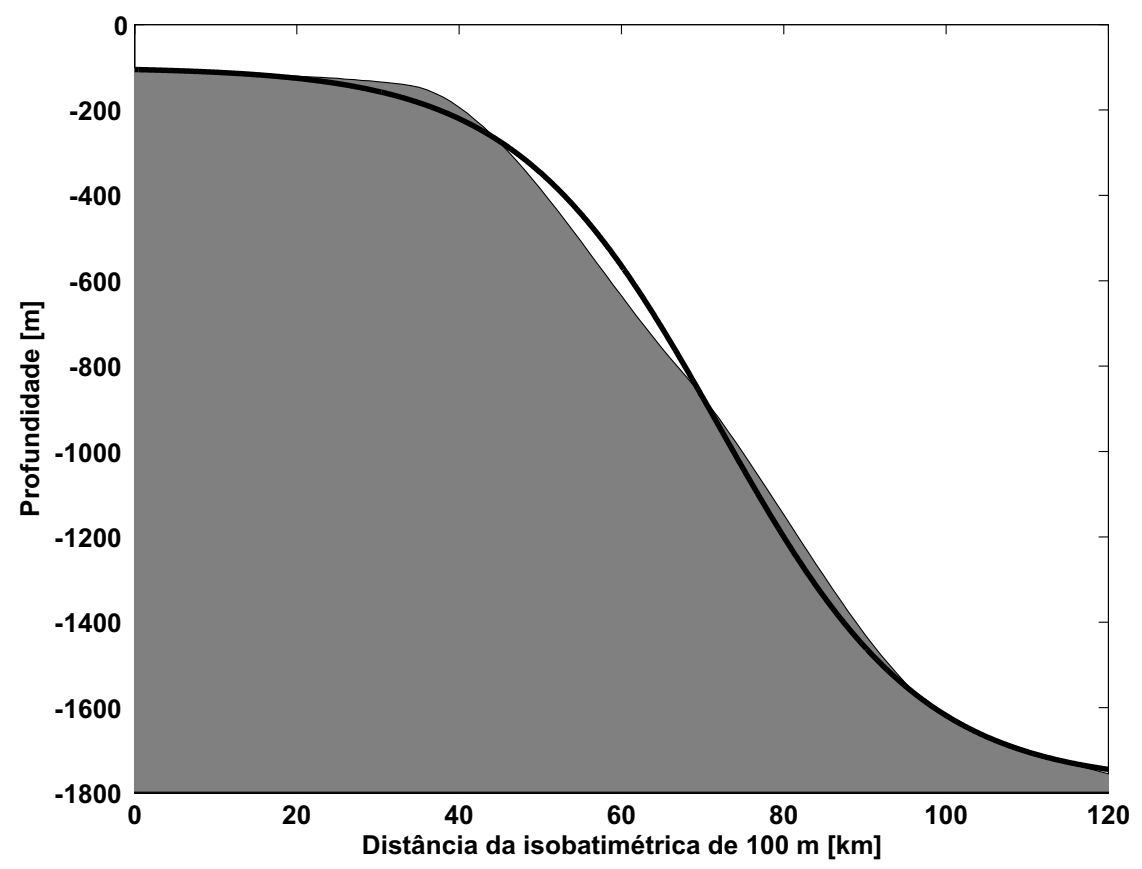

Figura 14 - Topografia real extraída dos dados do Cruzeiro DEPROAS (área em cinza) e analítica (linha negra) na seção em 23오.

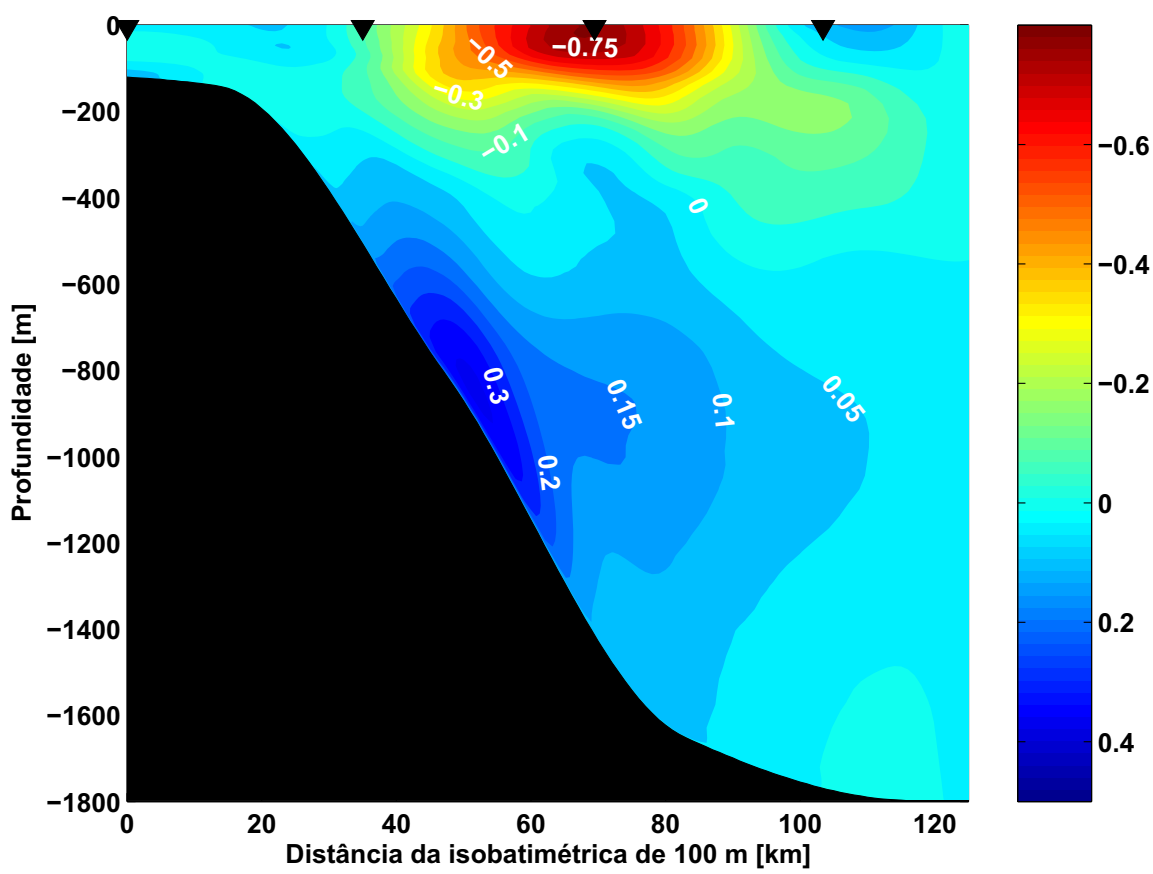

Figura 15 - Seção de velocidade baroclínica em $\mathrm{m} \mathrm{s}^{-1}$ simulada pelo modelo POMsec, inicializado com perfis de CTD do Projeto DEPROAS (julho de 2001), seguindo procedimentos apresentados por Silveira et al. (2004). Velocidades negativas têm a direção sul-sudoeste, e positivas norte-nordeste. 

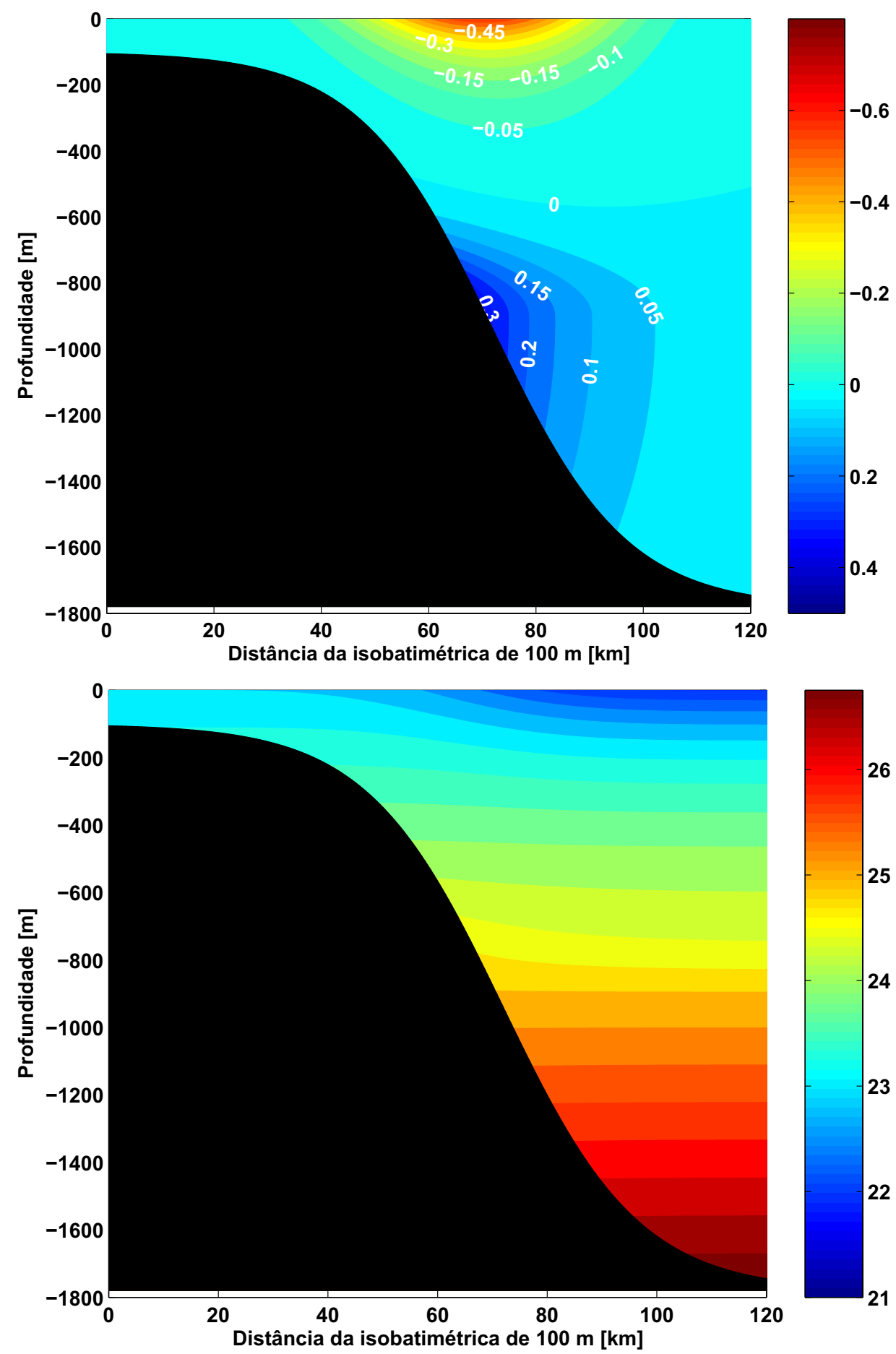

Figura 16 - Estado básico do Sistema CB, representativo de uma seção transversal na latitude de $23^{\circ} \mathrm{S}$ : campo de velocidade geostrófica em $\mathrm{m} \mathrm{s}^{-1}$ (painel superior) e campo de densidade $\sigma_{t} \mathrm{em} \mathrm{kg} \mathrm{m}^{-3}$ (painel inferior). Velocidades negativas têm a direção sul-sudoeste, e positivas norte-nordeste. 
na qual uma forma linearizada da equação de estado da água do mar é utilizada para a obtenção de um campo parametrizado de temperatura ajustado geostroficamente.

Em relação a outras aplicações, encontra-se em desenvolvimento um modelo de instabilidade linear, baseado em Xue \& Mellor (1993), que usa como campos de entrada as seções de velocidade e densidade básica desenvolvidas no presente artigo.

\section{REFERÊNCIAS}

ARANGO HA, GANGOPADHYAY A \& ROBINSON AR. 1992. Feature Models for the Western North Atlantic: Currents, Subbasin, and Mesoscale Features in the GSMR Region. Harvard University, Rep. in Meteorology and Oceanography 43. $60 \mathrm{pp}$.

BOEBEL 0, DAVIS RE, OLLITRAUT M, PETERSON R, RICHARD P, SCHMID C \& ZENK W. 1999. The Intermediate Depth Circulation of the Western South Atlantic, Geophys. Res. Let., 26(21): 3329-3332.

CUSHMAN-ROISIN B. 1994. Introduction to Geophysical Fluid Dynamics. Prentice-Hall, Inc., 320 pp.

FERNANDES FPA. 2005. Modelos Teóricos Paramétricos do Sistema Corrente do Brasil. Tese de Mestrado, Instituto Oceanográfico, Universidade de São Paulo, 71 pp.

GANGOPADHYAY A \& ROBINSON AR. 2002. Feature-oriented regional modeling of oceanic fronts. Dynam. Atmos. Oceans, 36: 201-232.

GANGOPADHYAY A, ROBINSON AR \& ARANGO HG. 1997. Circulation and Dynamics of the Western North Atlantic. Part I: Multiscale Feature Models. J. Atmos. Ocean. Tech., 14: 1314-1332.

LUTHER ME \& BANE JM. 1985. Mixed Instabilities in the Gulf Stream over the Continental Slope. J. Phys. Oceanogr., 15(1): 3-23.

NIILER PP \& ROBINSON AR. 1967. The Theory of free inertial jets: II. A Numerical Experiment for the Path of the Gulf stream. Tellus, 19(4): 601-619.

PEDLOSKY J. 1987. Geophysical Fluid Dynamics. Springer, New York, $710 \mathrm{pp}$.

ROBINSON AR, SPALL MA \& PINARDI N. 1988. Gulf Stream Simulations and the Dynamics of Ring and Meander Processes. J. Phys. Oceanogr., 18(12): 1811-1854.

SILVEIRA ICA, CALADO L, CASTRO BM, CIRANO M \& LIMA JAM. 2004.

On the baroclinic structure of the Brazil Current-Intermediate western Boundary Current system at $22^{\circ}-23^{\circ} \mathrm{S}$. Geophys. Res. Let., 31 : 4308 4312.

SILVEIRA ICA, SCHMIDT ACK, CAMPOS EJD, GODOI SSD \& IKEDA Y. 2000. A Corrente do Brasil ao Largo da Costa Leste Brasileira. R. Bras. Oceanogr., 48(2): 171-183.

STRAMMA L \& ENGLAND M. 1999. On the water masses and mean circulation of the South Atlantic Ocean. J. Geophys. Res., 104: 20.86320.884 .

XUE H \& MELLOR G. 1993. Instability of the Gulf Stream Front in the South Atlantic Bight. J. Phys. Oceanogr., 23: 2326-2350.

\section{NOTAS SOBRE OS AUTORES}

André Campos Kersten Schmidt. Bacharel em física e mestre em física teórica pela Universidade Federal de Santa Catarina (UFSC). Doutor em Oceanografia Física pelo Instituto Oceanográfico da Universidade de São Paulo (IOUSP), com estágio de um ano e meio no Department of Earth, Atmospheric and Planetary Sciences (EAPS), pertencente ao Massachusetts Institute of Technology (MIT), EUA. Atualmente, é Pós-doutorando no IOUSP. Especialista em Dinâmica de Contornos (DC), atua na área de dinâmica de fluidos geofísicos, aplicada aos oceanos, utilizando modelos matemáticos analíticos e numéricos. É colaborador em diversos projetos científicos nacionais e internacionais.

Wellington Ceccopieri Belo. Formado em Eletrônica (CEFET-RJ/1988), é graduado em Oceanografia (UERJ/1998), e mestre em Geologia/Geofísica Marinha (LAGEMAR-UFF-ANP/2001). Tem atuado nas áreas de Oceanografia Física, Meteo-Oceanografia e Oceanografia Geológica. É Oceanógrafo do Centro de Pesquisas da PETROBRAS (CENPES), onde colabora na investigação observacional de vórtices e meandros do Sistema Corrente do Brasil (CB/CCI), junto ao IO-USP, e no desenvolvimento de parâmetros de projeto para unidades marinhas de produção de óleo e gás. Seus interesses de pesquisa envolvem dinâmica de sedimentos, circulação oceânica de meso e grande escalas e dinâmica do escoamento da Corrente do Brasil.

Ilson Carlos Almeida da Silveira. Professor Doutor do Departamento de Oceanografia Física do Instituto Oceanográfico da Universidade de São Paulo (IOUSP), onde vem lecionando e desenvolvendo pesquisas desde 1997, sendo orientador de três doutorandos, cinco mestrandos e quatro alunos de iniciação científica, além de ter formado três doutores e cinco mestres. Graduado em Oceanografia pela Universidade do Estado do Rio de Janeiro (UERJ) em 1984, Mestre em Oceanografia Física pelo IOUSP em 1990 e Doutor pela University of New Hampshire em 1996, fez seu pós-doutoramento em Rhode Island no Graduate School of Oceanography (GSO), entre 1996 e 1997. Desde então, participou de vários projetos de pesquisa na qualidade de responsável e colaborador. Sua linha de pesquisa compreende a circulação de meso e larga escalas do oceano, voltada para teoria de instabilidade hidrodinâmica e para o estudo da dinâmica das correntes de contorno oeste, principalmente da Corrente do Brasil, através do desenvolvimento e utilização de modelos analíticos, semi-analíticos e numéricos.

José Antonio Moreira Lima. Consultor Sênior da PETROBRAS, trabalhando no grupo de Oceanografia Física da gerência de Métodos Científicos do Centro de Pesquisas e Desenvolvimento Leopoldo A. Miguez de Mello. Graduado em Engenharia Mecânica pela Universidade Federal da Bahia em 1983, tornou-se mestre em Engenharia Oceânica em 1986 pela COPPE/UFRJ. Obteve doutorado em Oceanografia Física pelo Departamento de Matemática Aplicada da Universidade de New South Wales (Austrália) em 1997. Tem se dedicado a estudos observacionais e numéricos de correntes de contorno oeste ao largo da costa brasileira com aplicações à exploração de petróleo em águas profundas. 